\title{
Regional Differences and Countermeasures of Low Carbon Economy Development in China
}

\author{
Yin Long \\ School of Economics, Harbin University of Commerce, Harbin, 150028, China
}

Keywords: low carbon economy; regional economy; carbon emissions; coordinated development

\begin{abstract}
In the process of world economic development, the environment and resources are becoming more and more prominent, the economic development mode is changing urgently, and the low-carbon development model emerges as the times require. Affected by the development pressure at home and abroad, a low-carbon economy is imperative in China. However, at present, the level of development of low-carbon economy among regions in China is quite different, which is not conducive to the sustainability of low-carbon development. In order to realize the coordination of China's low-carbon economic development, this paper analyzes the regional differences and causes of China's low-carbon economy, and proposes the development path of regional low-carbon economy in China.
\end{abstract}

\section{Introduction}

The negative effects of the rapid economic development are becoming more and more serious, and environmental protection issues have attracted much attention. The increase in carbon dioxide emissions has led to global warming, which was discovered by scientists as early as the end of the nineteenth century. The pioneer of the industrial revolution, in the course of its industrial economy, gradually realized the lack of natural resources and the pressure of the environment, and first proposed the concept of a low-carbon economy. Governments are also continually aware that the continued growth of carbon dioxide emissions will have a negative impact on the global environment, which in turn will lead to a global economic downturn. Therefore, the path of low carbon development has become the consensus of economic development in all countries of the world. The Jingdong Protocol, which came into effect in 2005, the United Nations Climate Conference in Copenhagen in 2009, and the arrival of the 2015 Paris Agreement have witnessed the determination of the world to reduce carbon emissions and protect the environment. After years of hard work, the global low-carbon economy has achieved initial success. According to the report released by the United Nations Panel of Climate Experts in 2017, carbon emissions have been in operation for three consecutive years in 2014-2016, without significant changes in global economic growth. The decline has shown a good trend of decoupling carbon emissions from economic growth.

China's economy has achieved remarkable results in the past 30 years, but the environmental problems caused by it have become more serious, which has caused serious negative impacts on the global climate and China's natural environment. The conflict between China's economic development and the environment has become more and more obvious, and environmental problems have become one of the important factors restricting economic development. When China's economy has not yet become the largest economic power, China has become a veritable "super carbon emitter". In 2004, China's carbon emissions exceeded that of the European Union. In 2007, it surpassed the United States to become the world's number one carbon emitter. Constrained by global warming, energy crisis and China's economic development model, China's economic development has slowed down significantly in recent years. In order to cope with this reality, it is imperative to abandon the "three highs and one low" extensive economic development model and take the "low carbon" development path. As a responsible big country, China has been actively responding to global warming in recent years, achieving the goal of $20 \%$ energy conservation and emission reduction during the 11th 
Five-Year Plan period, and promising China's GDP per unit of carbon emissions in 2020. It is 40\%-45\% lower than 2005. Under the Paris Agreement, which was launched in 2016, China promised to peak its carbon emissions in 2030 and achieve a 60\%-65\% reduction in carbon emissions per unit of GDP.

As the world's second largest economy, China has become the leader of global low-carbon economic development. However, due to resource endowments and historical reasons, China's low-carbon economy has different levels of development in different regions. This situation is not conducive to China's low carbon. Long-term economic development. It is worthwhile to study the reasons for the imbalance of low-carbon development in China's region and to develop targeted low-carbon development strategies based on regional differences.

\section{Regional differences in low-carbon economic development and analysis of influencing factors}

\subsection{Regional differences in low carbon economic development}

According to the level of regional low-carbon economy development in China, the development of low-carbon economy can be roughly divided into the following four categories: high and low carbon economy, higher low carbon economy, medium and low carbon economy and low carbon economy. The regions of high and low carbon economy include Beijing, Shanghai, Tianjin, Guangdong and Zhejiang. In the development of low carbon economy, the development level of Beijing, Shanghai and Tianjin is relatively good. All three cities are municipalities, economy and society. The environment and technology are at the forefront of the country and have good basic conditions. Although it performs poorly in terms of pollution emissions and pollution control, it does not affect the overall low carbon economy development level. The reason why Guangdong and Zhejiang are at the first level is that Guangdong has a strong economic comprehensive strength and has become a pilot province for low-carbon economy earlier; Zhejiang has been committed to industrial upgrading and industrial restructuring for many years. Green development path.

The regions with higher and lower carbon economy include Jiangsu, Fujian, Shandong, Hubei, Hunan and other regions. Jiangsu and Fujian have earlier understanding of carbon emissions and have been involved in environmental protection projects earlier; Shandong Province has attached importance to the development of low-carbon economy, established a low-carbon economic development promotion center, and actively introduced and utilized low-carbon technologies; Inner Mongolia In recent years, it has achieved fruitful results in the fields of afforestation and grassland protection. The good development of the environment has improved its low-carbon economy. Hubei Province has focused on low-carbon economic development, formulated low-carbon development plans and established a carbon emission trading center. Has achieved certain results.

The medium and low carbon economic development areas include Jiangxi, Liaoning, Anhui, Jilin, and Hebei. The Jiangxi region is mainly unreasonable in economic structure, and the low-carbon technology level is low, resulting in a low level of low-carbon economic development. Jilin and Liaoning are high-carbon emissions due to unreasonable industrial structure, but their land area is broad and carbon. The situation of foreign exchange is better, and its low-carbon economy is improved; Henan and Anhui are mainly low-level economic development and unreasonable economic structure; Hebei is affected by the transfer of Beijing-Tianjin industry, and various aspects need to be improved.

Low and low carbon economic development zones include Guizhou, Sichuan, Heilongjiang, Xinjiang, and Shanxi. The level of economic development in Guizhou is low and the level of low-carbon economy technology is backward. The Heilongjiang region is China's heavy industry base. The unreasonable industrial structure has inevitably become a hard-hit area for carbon emissions. The development of low-carbon economy in Xinjiang and Gansu started late. The relevant measures are mainly carried out after 2015, and the implementation of the measures has a certain lag to its actual effect; the low energy efficiency in Shanxi is the most important factor affecting the 
development of its low-carbon economy. The unique coal resources have not been well received. Utilization has become the most prominent problem.

\subsection{Factors affecting the development of low carbon economy}

\subsubsection{Differences in economic development levels}

The level of economic development will directly affect the low-carbon economy. The level of economic development and the level of low-carbon economy should be positively correlated. The higher the level of economic development, the higher the level of low-carbon economy, and vice versa. The level of economic development includes economic growth and structural adjustment. To develop a low-carbon economy, we must pay attention to the quality of economic development, and we need to transform the extensive economic development model and reduce the carbon emissions per unit of GDP. The difference in economic development levels across regions is one of the main reasons for regional differences in the carbon economy.

\subsubsection{Environmental differences}

The development of low-carbon economy is directly affected by environmental changes, the ecological environment is developing in a good direction, and the low-carbon economy is naturally in a positive direction. The environmental differences between regions are mostly natural. The forest coverage, human grassland area and per capita wetland area are difficult to change much in a short period of time. However, the regional environmental level is not static and is a dynamic process. The innate natural environment does not mean that the natural environment is also poor in the later period. Good protection policies and actions can continuously improve the regional natural environment level, and unreasonable environmental development strategies may also lead to deterioration of the natural environment.

\subsubsection{Differences in energy consumption}

Energy consumption is one of the main factors affecting carbon emission levels. As energy consumption increases, carbon emissions also increase, which has a negative effect on the development of low-carbon economy. Energy consumption is the main source of regional carbon emissions, and the greater the energy consumption, the greater the negative impact on climate and the environment. The difference in energy consumption across regions generally covers both energy consumption and energy consumption structure. Affected by the level of regional economic development, the composition of industrial structure, and the different structures of industrial energy consumption and domestic energy consumption, the total energy consumption of different regions is different, and the impact on the development of low-carbon economy is also very different.

\subsubsection{Differences in technology levels}

Science and technology are the primary productive forces, and the level of low-carbon technology directly determines the level of development of the low-carbon economy. Technological innovation can drive the transformation of productivity, the problem of "carbon pollution" will be better solved, and the development of low-carbon economy will be promoted. In the field of production, low-carbon technology can effectively improve the efficiency of energy use and directly reduce carbon emissions. In the field of low-carbon energy, the development and utilization of new energy sources such as solar energy and tidal energy enhances the utilization level of clean energy and enables energy structure. It tends to be reasonable; in the field of carbon emission control, low-carbon technology can improve resource utilization efficiency, reduce unnecessary waste, and achieve harmonious development of economy and environment. However, there are obvious differences in the technical level between regions in China, and there are certain obstacles to the dissemination of innovative technologies. 


\section{Regional low carbon economy differential development path}

\subsection{Reduce regional differences and achieve coordinated development of low-carbon economy}

Changing the status quo of regional differences in China's low-carbon economy development is a long-term and arduous task that requires the state to carry out regulation and control at a global level. In narrowing regional differences and realizing the all-round development of a low-carbon economy, it is necessary to properly handle the interests of the state, local governments, and people. On the one hand, the development of regional low-carbon economy needs the guidance and support of the state. At the same time, it must also pay attention to the interests of local governments, grasp the problems or new requirements of local governments in the process of low-carbon economy development, and take into account the fundamental interests of the people in the region. Make corresponding adjustments to the low carbon economic development policy. For regions with low and low carbon economic development, the state should increase its support. These regions have made great economic sacrifices for the national ecological environment security. As compensation, industries that are favorable to the development of the western region should be given preferential tax policies.

\subsection{Combine regional characteristics and adopt a reasonable development strategy}

The imbalance of low-carbon economic development in China's regions is an objective reality, but the reasons for the imbalance are multi-faceted. According to the respective characteristics of different regions, a reasonable development strategy should be adopted. For higher and lower carbon development areas, we should continue to explore the potential of low-carbon resources development, vigorously develop low-carbon industries, and enhance independent innovation capabilities. For medium and low carbon development areas, the overall economic level should be improved, the benefits of natural resource development should be improved, the industrial agglomeration effect should be emphasized, and the industrial structure and energy consumption structure should be optimized. For areas with low and low carbon economy development, we should vigorously improve the level of economic development, improve resource utilization, focus on ecological environment construction, and develop local specialty industries.

\subsection{Encourage independent innovation and technology development, and strengthen inter-regional exchanges and cooperation}

Innovation is the key to improving the level of science and technology and economic competitiveness. Higher independent innovation ability can effectively ensure the transformation of economic growth mode and industrial restructuring. China should improve the mechanism of scientific and technological innovation, enhance the strength of technological innovation, enhance the level of independent innovation, and serve the development of low-carbon economy. In the aspect of low-carbon emission reduction technologies, research and development of energy conservation technologies should be strengthened, energy conservation and consumption reduction technologies should be researched and promoted, resource interest rates should be raised, pollution emissions should be reduced; research and development of clean energy and renewable energy technologies should be strengthened, and organisms should be developed. High-tech projects such as engineering, new materials and oceans reduce the dependence of industrial production on non-renewable resources; strengthen the research and development of environmental pollution control and ecological comprehensive management technologies. At the same time, it pays attention to exchanges and cooperation between regions, realizes technology sharing, and maximizes the benefits of scientific and technological achievements.

\section{References}

[1] Hu Angang, Guan Qingyou. Four Feasibility of China's Response to Global Climate Change [J]. Journal of Tsinghua University (Philosophy and Social Sciences), 2008(6) 
[2] Zhang Hongfeng, Zhou Feng, Yang Hui, Guo Qing. An Empirical Analysis of the Performance of Environmental Protection and Economic Development in a Win-win Situation [J]. Economic Research, 2009(3)

[3] Chen Shiyi. Evaluation of the transition process of low-carbon economy in various regions of China [J]. Economic Research, 2012 (8)

[4] Jia Dengxun, Huang Jie. Regional Differences and Influencing Factors of Low Carbon Economy Development Efficiency [J]. Journal of Lanzhou University (Social Science Edition), 2014(7). 